\title{
Weighted Hardy's inequality in a limiting case and the perturbed Kolmogorov equation
}

Megumi Sano \& Futoshi Takahashi

\begin{tabular}{|c|l|}
\hline Citation & Applicable Analysis. 98(10); $1875-1888$. \\
\hline Issue Date & $2018-05-28$ \\
\hline Type & Journal Article \\
\hline Textversion & author \\
\hline Rights & $\begin{array}{l}\text { This is an Accepted Manuscript of an article published by Taylor \& Francis in } \\
\text { Applicable Analysis on } 28 / 5 / 2018, \text { available online: } \\
\text { https://doi.org/10.1080/00036811.2018.1471208. }\end{array}$ \\
\hline DOI & $10.1080 / 00036811.2018 .1471208$ \\
\hline
\end{tabular}

\author{
Self-Archiving by Author(s) \\ Placed on: Osaka City University
}




\title{
Weighted Hardy's inequality in a limiting case and the perturbed Kolmogorov equation
}

\author{
Megumi Sano - Futoshi Takahashi
}

November 12, 2019

\begin{abstract}
In this paper, we show a weighted Hardy inequality in a limiting case for functions in weighted Sobolev spaces with respect to an invariant measure. We also prove that the constant in the left-hand side of the inequality is optimal. As applications, we establish the existence and nonexistence of positive exponentially bounded weak solutions to a parabolic problem involving the Ornstein-Uhlenbeck operator perturbed by a critical singular potential in two dimensional case, according to the size of the coefficient of the critical potential. These results can be considered as counterparts in the limiting case of results which established in [8] [10] in the non-critical cases, and are also considered as extensions of a result in [4] to the Kolmogorov operator case perturbed by a critical singular potential.
\end{abstract}

Keywords Weighted Hardy's inequality · Limiting case · Kolmogorov operator · Singular potential

Mathematics Subject Classification (2010) 35 A23 $\cdot 26$ D 10.

\section{Introduction}

Let $\Omega$ be a domain in $\mathbb{R}^{N}$ with $0 \in \Omega, N \geq 1,1<p<\infty, A$ be a real $N \times N$-symmetric positive semi-definite matrix, and

$$
d \mu_{A}=\rho_{A}(x) d x \quad \text { with } \quad \rho_{A}(x)=c \cdot \exp \left(-\frac{1}{p}\left(x^{t} A x\right)^{\frac{p}{2}}\right), x \in \Omega .
$$

Megumi Sano

Department of Mathematics, Tokyo Institute of Technology, Meguro-ku, Tokyo, 152-8550, Japan

E-mail: sano.m.af@m.titech.ac.jp \& megumisano0609@gmail.com

Futoshi Takahashi

Department of Mathematics, Graduate School of Science \& OCAMI, Osaka City University, 3-3-138

Sugimoto, Sumiyoshi-ku, Osaka, 558-8585, Japan

E-mail: futoshi@sci.osaka-cu.ac.jp 
Here $c>0$ is chosen so that $\int_{\Omega} d \mu_{A}=1$. More generally, we consider a Borel probability measure $d \mu=\rho(x) d x$ defined on $\Omega \subseteq \mathbb{R}^{N}$, and let $W_{\mu, 0}^{1, p}(\Omega)$ denote a weighted Sobolev space which is a completion of $C_{c}^{\infty}(\Omega)$ with respect to the (semi-) norm $\|\nabla \cdot\|_{L^{p}(\Omega ; d \mu)}$.

In this paper, we concern the limiting case $p=N \geq 2$. Let $\Omega \subset \mathbb{R}^{N}$ be a bounded domain with $R=\sup _{x \in \Omega}|x|<\infty$ and $a \geq 1$. Let $p=N \geq 2$ in (1). In this paper, first we show the following weighted critical Hardy inequality

$$
\begin{aligned}
\left(\frac{N-1}{N}\right)^{N} \int_{\Omega} \frac{|u|^{N}}{|x|^{N}\left(\log \frac{a R}{|x|}\right)^{N}} d \mu_{A} & \leq \int_{\Omega}\left|\nabla u \cdot \frac{x}{|x|}\right|^{N} d \mu_{A} \\
& +\left(\frac{N-1}{N}\right)^{N-1} \int_{\Omega} \frac{|u|^{N}\left(x^{t} A x\right)^{\frac{N}{2}}}{|x|^{N}\left(\log \frac{a R}{|x|}\right)^{N-1}} d \mu_{A}
\end{aligned}
$$

holds for all $u$ in $W_{\mu_{A}, 0}^{1, N}(\Omega)$. We also prove that the constant $\left(\frac{N-1}{N}\right)^{N}$ in the left-hand side is optimal when $0 \in \Omega$. For a general weight function $\rho=\rho(x)$ satisfying some assumptions, we also prove a weighted critical Hardy inequality (with non-optimal constant) on two-dimensional domain.

The limiting case is left to be considered in [8] and [10]. Actually in [10], the authors prove a (non-critical) weighted Hardy inequality

$$
\begin{aligned}
\left(\frac{|N-p|}{p}\right)^{p} \int_{\Omega} \frac{|u|^{p}}{|x|^{p}} d \mu_{A} & \leq \int_{\Omega}\left|\nabla u \cdot \frac{x}{|x|}\right|^{p} d \mu_{A} \\
& +\left(\frac{|N-p|}{p}\right)^{p-1} \operatorname{sgn}(N-p) \int_{\Omega} \frac{|u|^{p}\left(x^{t} A x\right)^{\frac{p}{2}}}{|x|^{p}} d \mu_{A}
\end{aligned}
$$

for functions $u$ in $W_{\mu_{A}, 0}^{1, p}(\Omega)$ when $1<p<N, N \geq 2$, and $u$ in $W_{\mu_{A}, 0}^{1, p}(\Omega \backslash\{0\})$ when $p>N \geq 1$, where $\mu_{A}$ is defined in (1). The inequality (3) was first established in [8] when $p=2$ and $N \geq 3$.

Next, by using the optimality of the critical Hardy constant for $\rho_{A}$ with $p=N$, we study the existence and nonexistence of positive weak solutions of a parabolic equation driven by the symmetric Ornstein-Uhlenbeck operator perturbed by a singular potential in dimension $N=2$. This part can be considered as an extension of [8] to the two dimensional critical case. Indeed, by using the weighted Hardy inequality (3) for $\mu_{A}$ with $p=2$ and $N \geq 3$, and a result similar to the one in [4] which is applicable to the Kolmogorov operator

$$
L u=\Delta u+\frac{\nabla \rho}{\rho} \cdot \nabla u
$$

with respect to a positive Borel probability measure $d \mu=\rho d x$, the authors in [8] prove the following result.

Theorem 1 (Goldstein-Goldstein-Rhandi [8]) Assume $N \geq 3$ and $p=2$ in (1). Let $A$ be a real $N \times N$-symmetric positive definite matrix and $0 \leq V(x) \leq \frac{c}{|x|^{2}}, x \in \mathbb{R}^{N}$. Then the following assertions hold: 
of

(i) If $0 \leq c \leq\left(\frac{N-2}{2}\right)^{2}$, then there exists a weak solution $u \in C\left([0, \infty), L^{2}\left(\mathbb{R}^{N} ; d \mu_{A}\right)\right)$

$$
\begin{cases}\partial_{t} u(x, t)=\Delta u(x, t)-A x \cdot \nabla u(x, t)+V(x) u(x, t), & t>0, x \in \mathbb{R}^{N}, \\ u(x, 0)=u_{0}(x), & x \in \mathbb{R}^{N},\end{cases}
$$

satisfying

$$
\|u(t)\|_{L^{2}\left(\mathbb{R}^{N} ; d \mu_{A}\right)} \leq M e^{\omega t}\left\|u_{0}\right\|_{L^{2}\left(\mathbb{R}^{N} ; d \mu_{A}\right)}, \quad t \geq 0
$$

for some constants $M \geq 1, \omega \in \mathbb{R}$ and for any $0 \leq u_{0} \in L^{2}\left(\mathbb{R}^{N} ; d \mu_{A}\right)$.

(ii) If $c>\left(\frac{N-2}{2}\right)^{2}$, then for any $0 \leq u_{0} \in L^{2}\left(\mathbb{R}^{N} ; d \mu_{A}\right) \backslash\{0\}$, there is no positive weak solution with $V(x)=\frac{c}{|x|^{2}}$ satisfying the above exponential boundedness.

Note that if $\mu=\mu_{A}$ with $p=2$, then the Kolmogorov operator $L$ is of the form

$$
L_{A} u=\Delta u-A x \cdot \nabla u,
$$

which is known as the symmetric Ornstein-Uhlenbeck operator. This type of operator arises from many areas of mathematics, such as probability, mathematical physics, and mathematical finance. Later, Theorem 1 was generalized by Hauer-Rhandi [10] to the case $p \neq 2$ and $\Omega=(0, \infty) \subset \mathbb{R}(N=1)$ and Goldstein-Hauer-Rhandi [9] for the general case. See also [7]. For the classical case $L=\Delta$, the study of existence and nonexistence of positive solutions to the heat equation with a singular potential was initiated by Baras-Goldstein [3] and now enjoys various extensions, see [2], [5], [6], [11], [12], and the references therein.

In this paper, we prove the corresponding result for the parabolic problem driven by the symmetric Ornstein-Uhlenbeck operator perturbed by a singular critical potential of the form $V(x)=\frac{c}{|x|^{2}\left(\log \frac{a R}{|x|}\right)^{2}}$ on two dimensional bounded domains with the Dirichlet boundary conditions.

At the end of this section, we fix several notations: Let $B^{k}(R)$ be the $k$-dimensional ball centered at the origin with radius $R$ in $\mathbb{R}^{k} . B^{N}(R)$ will be denoted by $B(R) .\left|B^{k}(R)\right|$ denotes the $k$-dimensional volume of $B^{k}(R) . \omega_{N}$ denotes the area of the unit sphere in $\mathbb{R}^{N} .(X)_{N}$ be the $N$-th component of the vector $X \in \mathbb{R}^{N}$.

\section{A weighted critical Hardy inequality : $p=N$}

In this section, we prove several weighted Hardy type inequalities for functions in the critical weighted Sobolev space. Next theorem is a generalization of a result in [10] to the critical case. Critical Hardy type inequalities with sharp $(a=1)$ or non-sharp $(a>1)$ weight when $d \mu$ is the Lebesgue measure have been studied by many authors recently, see for example, [1], [13], [16], [17], [18], [19] and the references therein. The sharp critical Hardy inequality was proved originally by Leray in his thesis in 1933 [14] when $N=2$. 
Theorem 2 Let $\Omega$ be a bounded domain in $\mathbb{R}^{N}, N \geq 2, R=\sup _{x \in \Omega}|x|, a \geq 1$, and $A$ be a real $N \times N$-symmetric positive semi-definite matrix. Let $\mu_{A}$ be defined in (1) with $p=N$. Then the inequality

$$
\begin{aligned}
\left(\frac{N-1}{N}\right)^{N} \int_{\Omega} \frac{|u|^{N}}{|x|^{N}\left(\log \frac{a R}{|x|}\right)^{N}} d \mu_{A} & \leq \int_{\Omega}\left|\nabla u \cdot \frac{x}{|x|}\right|^{N} d \mu_{A} \\
& +\left(\frac{N-1}{N}\right)^{N-1} \int_{\Omega} \frac{|u|^{N}\left(x^{t} A x\right)^{\frac{N}{2}}}{|x|^{N}\left(\log \frac{a R}{|x|}\right)^{N-1}} d \mu_{A}
\end{aligned}
$$

holds for all $u \in W_{\mu_{A}, 0}^{1, N}(\Omega)$. Moreover, if $0 \in \Omega$, then the constant $\left(\frac{N-1}{N}\right)^{N}$ in the lefthand side of (4) is optimal.

Proof of Theorem 2. By density, it is enough to show that the inequality (4) holds for all $u \in C_{c}^{1}(\Omega)$. We fix $\lambda \geq 0$ and $\beta>0$, which will be chosen later. Set

$$
\mathbf{F}(x)=\lambda \rho_{A}(x) \frac{x}{|x|^{N}\left(\log \frac{a R}{|x|}\right)^{\beta}} \quad \text { for } x \in \Omega \backslash\{0\} .
$$

Here $\rho_{A}$ is defined in (1) with $p=N$. Then we easily check that

$$
\operatorname{div} \mathbf{F}(x)=\lambda \rho_{A}(x)\left[\frac{\beta}{|x|^{N}\left(\log \frac{a R}{|x|}\right)^{\beta+1}}-\frac{\left(x^{t} A x\right)^{\frac{N}{2}}}{|x|^{N}\left(\log \frac{a R}{|x|}\right)^{\beta}}\right] \quad \text { for } x \in \Omega \backslash\{0\} .
$$

By applying integration by parts and Young's inequality, we have

$$
\begin{aligned}
& \int_{\Omega}|u|^{N} \lambda\left[\frac{\beta}{|x|^{N}\left(\log \frac{a R}{|x|}\right)^{\beta+1}}-\frac{\left(x^{t} A x\right)^{\frac{N}{2}}}{|x|^{N}\left(\log \frac{a R}{|x|}\right)^{\beta}}\right] d \mu_{A} \\
& =-N \int_{\Omega}|u|^{N-2} u(\nabla u \cdot \mathbf{F}) d x \\
& =-N \lambda \int_{\Omega} \frac{|u|^{N-2} u}{|x|^{N-1}\left(\log \frac{a R}{|x|}\right)^{\beta}}\left(\nabla u \cdot \frac{x}{|x|}\right) d \mu_{A} \\
& \leq \int_{\Omega}\left|\nabla u \cdot \frac{x}{|x|}\right|^{N} d \mu_{A}+(N-1) \lambda^{\frac{N}{N-1}} \int_{\Omega} \frac{|u|^{N}}{|x|^{N}\left(\log \frac{a R}{|x|}\right)^{\frac{\beta N}{N-1}}} d \mu_{A} .
\end{aligned}
$$

Here note that the left-hand side of the first equality is well-defined because the following properties hold true by the assumption of $A$ :

$$
\frac{1}{|x|^{N}\left(\log \frac{a R}{|x|}\right)^{\beta+1}} \in L_{\mathrm{loc}}^{1}(\Omega) \quad \text { and } \quad \frac{\left(x^{t} A x\right)^{\frac{N}{2}}}{|x|^{N}\left(\log \frac{a R}{|x|}\right)^{\beta}} \leq \frac{|A|^{\frac{N}{2}}}{\left(\log \frac{a R}{|x|}\right)^{\beta}} \in L_{\mathrm{loc}}^{1}(\Omega) .
$$

Now, if we choose $\beta=N-1$, then we obtain

$$
\begin{aligned}
\left(\lambda(N-1)-(N-1) \lambda^{\frac{N}{N-1}}\right) \int_{\Omega} \frac{|u|^{N}}{|x|^{N}\left(\log \frac{a R}{|x|}\right)^{N}} d \mu_{A} & \leq \int_{\Omega}\left|\nabla u \cdot \frac{x}{|x|}\right|^{N} d \mu_{A} \\
& +\lambda \int_{\Omega} \frac{|u|^{N}\left(x^{t} A x\right)^{\frac{N}{2}}}{|x|^{N}\left(\log \frac{a R}{|x|}\right)^{N-1}} d \mu_{A} .
\end{aligned}
$$


Furthermore, if we choose $\lambda=\left(\frac{N-1}{N}\right)^{N-1}$ which attains the maximum of the function $\lambda \mapsto\left(\lambda(N-1)-(N-1) \lambda^{\frac{N}{N-1}}\right)$ on the half line $[0, \infty)$, then we obtain the inequality (4) for all $u \in C_{c}^{1}(\Omega)$. Therefore the inequality (4) also holds for all $u \in W_{\mu_{A}, 0}^{1, N}(\Omega)$.

Next we shall show the optimality of the constant $\left(\frac{N-1}{N}\right)^{N}$ in (4) when $0 \in \Omega$. To do so, we fix $\lambda>\left(\frac{N-1}{N}\right)^{N}$ and take any $\tilde{\lambda} \geq 0$. Set

$$
\begin{aligned}
& E(u)=\int_{\Omega}\left|\nabla u \cdot \frac{x}{|x|}\right|^{N} d \mu_{A}+\tilde{\lambda} \int_{\Omega} \frac{|u|^{N}\left(x^{t} A x\right)^{\frac{N}{2}}}{|x|^{N}\left(\log \frac{a R}{|x|}\right)^{N-1}} d \mu_{A}, \\
& F_{\lambda}(u)=\lambda \int_{\Omega} \frac{|u|^{N}}{|x|^{N}\left(\log \frac{a R}{|x|}\right)^{N}} d \mu_{A}
\end{aligned}
$$

for $u \in W_{\mu_{A}, 0}^{1, N}(\Omega) \backslash\{0\}$. Now we consider the test function $\varphi_{\gamma, \varepsilon} \in W_{\mu_{A}, 0}^{1, N}(B(R))$ given by

$$
\varphi_{\gamma, \varepsilon}(x)=\left(\log \frac{a R}{|x|}\right)^{\gamma} \xi_{\varepsilon}(x)
$$

where $\gamma<\frac{N-1}{N}, \varepsilon>0$ is chosen so that $B(\varepsilon) \subset \Omega$, and $\xi_{\varepsilon} \in C_{c}^{\infty}(B(\varepsilon))$ is a cut-off function with $0 \leq \xi_{\varepsilon} \leq 1, \xi_{\varepsilon} \equiv 1$ on $B\left(\frac{\varepsilon}{2}\right),\left|\nabla \xi_{\varepsilon}\right| \leq B \varepsilon^{-1}$ on $B(\varepsilon)$ for some $B>0$. Note that there exists $\alpha_{2}>0$ such that $0 \leq x^{t} A x \leq \alpha_{2}|x|^{2}$ for all $x \in \Omega$, because $A$ is positive semi-definite. Then we have

$$
\begin{aligned}
F_{\lambda}\left(\varphi_{\gamma, \varepsilon}\right) & \geq \lambda \int_{B\left(\frac{\varepsilon}{2}\right)} \frac{\left|\varphi_{\gamma, \varepsilon}\right|^{N}}{|x|^{N}\left(\log \frac{a R}{|x|}\right)^{N}} \rho_{A}(x) d x \\
& \geq \lambda \int_{B\left(\frac{\varepsilon}{2}\right)}\left(\log \frac{a R}{|x|}\right)^{\gamma N-N} c \exp \left(-\frac{\alpha_{2}^{\frac{N}{2}}}{N}|x|^{N}\right) \frac{d x}{|x|^{N}} \\
& \geq \lambda c \exp \left(-\frac{\alpha_{2}^{\frac{N}{2}}}{N}\left(\frac{\varepsilon}{2}\right)^{N}\right) \omega_{N} \int_{0}^{\frac{\varepsilon}{2}}\left(\log \frac{a R}{r}\right)^{\gamma N-N} \frac{d r}{r} \\
& =\lambda c \exp \left(-\frac{\alpha_{2}^{\frac{N}{2}}}{N}\left(\frac{\varepsilon}{2}\right)^{N}\right) \frac{\omega_{N}}{N}\left(\log \frac{2 a R}{\varepsilon}\right)^{N\left(\gamma-\frac{N-1}{N}\right)}\left(\frac{N-1}{N}-\gamma\right)^{-1}
\end{aligned}
$$

And also we obtain

$$
\begin{aligned}
\int_{\Omega}\left|\nabla \varphi_{\gamma, \varepsilon} \cdot \frac{x}{|x|}\right|^{N} d \mu_{A} & \leq \gamma^{N} \int_{B\left(\frac{\varepsilon}{2}\right)}\left(\log \frac{a R}{|x|}\right)^{\gamma N-N} \rho_{A}(x) \frac{d x}{|x|^{N}}+c \int_{B(\varepsilon) \backslash B\left(\frac{\varepsilon}{2}\right)}\left|\nabla \varphi_{\gamma, \varepsilon}\right|^{N} d x \\
& \leq \gamma^{N} c \frac{\omega_{N}}{N}\left(\log \frac{2 a R}{\varepsilon}\right)^{N\left(\gamma-\frac{N-1}{N}\right)}\left(\frac{N-1}{N}-\gamma\right)^{-1}+R(\gamma, \varepsilon),
\end{aligned}
$$


where $R(\gamma, \varepsilon)=c \int_{B(\varepsilon) \backslash B\left(\frac{\varepsilon}{2}\right)}\left|\nabla \varphi_{\gamma, \varepsilon}\right|^{N} d x$. Note that the remainder term $R(\gamma, \varepsilon)$ can be estimated as follows:

$$
\begin{aligned}
& R(\gamma, \varepsilon) \\
& \leq c 2^{N-1} \int_{B(\varepsilon) \backslash B\left(\frac{\varepsilon}{2}\right)}\left|\xi_{\varepsilon} \nabla\left(\left(\log \frac{a R}{|x|}\right)^{\gamma}\right)\right|^{N}+\left|\left(\log \frac{a R}{|x|}\right)^{\gamma} \nabla \xi_{\varepsilon}\right|^{N} d x \\
& \leq c 2^{N-1} \gamma^{N} \int_{B(\varepsilon) \backslash B\left(\frac{\varepsilon}{2}\right)}\left(\log \frac{a R}{|x|}\right)^{(\gamma-1) N} \frac{d x}{|x|^{N}}+c 2^{N-1}\left(B \varepsilon^{-1}\right)^{N} \int_{B(\varepsilon) \backslash B\left(\frac{\varepsilon}{2}\right)}\left(\log \frac{a R}{|x|}\right)^{\gamma N} d x \\
& \leq c 2^{N-1} \omega_{N} \gamma^{N}((\gamma-1) N+1)^{-1}\left[\left(\log \frac{2 a R}{\varepsilon}\right)^{(\gamma-1) N+1}-\left(\log \frac{a R}{\varepsilon}\right)^{(\gamma-1) N+1}\right] \\
& \quad+c 2^{N-1} \omega_{N} B^{N}(\gamma N+1)^{-1}\left[\left(\log \frac{2 a R}{\varepsilon}\right)^{\gamma N+1}-\left(\log \frac{a R}{\varepsilon}\right)^{\gamma N+1}\right] .
\end{aligned}
$$

By applying the mean value theorem for the function $x \mapsto x^{p}$ for $p=(\gamma-1) N+1$ or $p=\gamma N+1$, there exist positive constants $b, d$ satisfying $\log \frac{a R}{\varepsilon} \leq b$ and $d \leq \log \frac{2 a R}{\varepsilon}$ such that

$$
\begin{aligned}
|R(\gamma, \varepsilon)| & \leq c 2^{N-1} \omega_{N} \log 2\left[\gamma^{N} b^{N\left(\gamma-\frac{N-1}{N}\right)-1}+B^{N} d^{\gamma N}\right] \\
& \leq c 2^{N-1} \omega_{N} \log 2\left[\gamma^{N}\left(\log \frac{a R}{\varepsilon}\right)^{N\left(\gamma-\frac{N-1}{N}\right)-1}+B^{N}\left(\log \frac{2 a R}{\varepsilon}\right)^{\gamma N}\right] \\
& =O(1) \quad \text { as } \gamma \nearrow \frac{N-1}{N} .
\end{aligned}
$$

In the same way as above, we also obtain

$$
\begin{aligned}
\int_{\Omega} \frac{\left|\varphi_{\gamma, \varepsilon}\right|^{N}\left(x^{t} A x\right)^{\frac{N}{2}}}{|x|^{N}\left(\log \frac{a R}{|x|}\right)^{N-1}} d \mu_{A} & \leq|A|^{\frac{N}{2}} c \omega_{N} \varepsilon^{N}(\gamma N-N+2)^{-1}\left(\log \frac{a R}{\varepsilon}\right)^{\gamma N-N+2} \\
& =O(1) \text { as } \gamma \nearrow \frac{N-1}{N} .
\end{aligned}
$$

From (6), (7), and (8), we have

$$
\begin{array}{rl}
E\left(\varphi_{\gamma, \varepsilon}\right) \leq \gamma^{N} & c \frac{\omega_{N}}{N}\left(\log \frac{2 a R}{\varepsilon}\right)^{N\left(\gamma-\frac{N-1}{N}\right)}\left(\frac{N-1}{N}-\gamma\right)^{-1} \\
+ & o\left(\left(\frac{N-1}{N}-\gamma\right)^{-1}\right) \text { as } \gamma \nearrow \frac{N-1}{N} .
\end{array}
$$

From the estimates (5) and (9), if we have chosen $\varepsilon>0$ independent of $\gamma$ so small such that $\lambda \exp \left(-\frac{\alpha_{2}^{\frac{N}{2}}}{N}\left(\frac{\varepsilon}{2}\right)^{N}\right)>\left(\frac{N-1}{N}\right)^{N}>\gamma^{N}$, which is possible since $\lambda>\left(\frac{N-1}{N}\right)^{N}$, then we observe that

$$
E\left(\varphi_{\gamma, \varepsilon}\right)<F_{\lambda}\left(\varphi_{\gamma, \varepsilon}\right)
$$

for $\gamma$ close to $\frac{N-1}{N}$. Therefore the inequality (4) never holds if the constant on the lefthand side of (4) is bigger than $\left(\frac{N-1}{N}\right)^{N}$. Hence the constant $\left(\frac{N-1}{N}\right)^{N}$ in (4) is optimal. 
Remark 1 Let $\tilde{\lambda} \geq 0$ and $\lambda>\left(\frac{N-1}{N}\right)^{N}$. Then by using the test function $\varphi_{\gamma, \varepsilon}$, we observe that

$$
\inf _{0 \neq u \in W_{\mu_{A}, 0}^{1, N}(\Omega)} \frac{\int_{\Omega}|\nabla u|^{N} d \mu_{A}+\tilde{\lambda} \int_{\Omega} \frac{|u|^{N}\left(x^{t} A x\right)^{\frac{N}{2}}}{|x|^{N}\left(\log \frac{a R}{|x|}\right)^{N-1}} d \mu_{A}-\lambda \int_{\Omega} \frac{|u|^{N}}{|x|^{N}\left(\log \frac{a R}{|x|}\right)^{N}} d \mu_{A}}{\int_{\Omega}|u|^{N} d \mu_{A}}=-\infty
$$

holds true.

In the case $N=2$, we can obtain the critical Hardy type inequality (with nonoptimal constant) for the general weight function $\rho=\rho(x)$ satisfying the following conditions:

$$
\begin{aligned}
& \text { (H1) } 0<\rho \in H^{2}(\Omega) \text {, } \\
& \text { (H2) for any } \varepsilon>0 \text { there is } C_{\varepsilon} \in \mathbb{R} \text { such that }\left|\frac{\nabla \rho}{\rho}\right|^{2}-\frac{\Delta \rho}{\rho} \leq \varepsilon\left|\frac{\nabla \rho}{\rho}\right|^{2}+C_{\varepsilon} \text {. }
\end{aligned}
$$

Proposition 1 Let $\Omega$ be a bounded domain in $\mathbb{R}^{2}$ containing the origin, $R=\sup _{x \in \Omega}|x|$ and $a \geq 1$. Let $d \mu=\rho(x) d x$ and assume (H1) and (H2) are satisfied. Then for any $\delta>0$, there exists $C_{\delta}>0$ such that the inequality

$$
\frac{1}{4} \int_{\Omega} \frac{|\phi|^{2}}{|x|^{2}\left(\log \frac{a R}{|x|}\right)^{2}} d \mu \leq(4+\delta) \int_{\Omega}|\nabla \phi|^{2} d \mu+C_{\delta} \int_{\Omega}|\phi|^{2} d \mu
$$

holds for all $\phi \in W_{\mu, 0}^{1,2}(\Omega)$.

Proof of Proposition 1. The proof goes along the same way as in [8]. Again we may assume $\phi \in C_{c}^{\infty}(\Omega)$. Since $\operatorname{div}\left(\frac{x}{|x|^{2} \log \frac{a R}{|x|}}\right)=\frac{1}{|x|^{2}\left(\left.\log \frac{a R}{|x|}\right|^{2}\right.}$, we obtain

$$
\begin{aligned}
\int_{\Omega} \frac{|\phi|^{2}}{|x|^{2}\left(\log \frac{a R}{|x|}\right)^{2}} d \mu & =\int_{\Omega}|\phi|^{2} \rho(x) \operatorname{div}\left(\frac{x}{|x|^{2}\left(\log \frac{a R}{|x|}\right)}\right) d x \\
& =-\int_{\Omega}\left(2 \phi \rho \nabla \phi+|\phi|^{2} \nabla \rho\right) \cdot \frac{x}{|x|^{2} \log \frac{a R}{|x|}} d x \\
& \leq 2 \int_{\Omega} \frac{|\phi|}{|x| \log \frac{a R}{|x|}\left|\nabla \phi+\frac{1}{2} \phi \frac{\nabla \rho}{\rho}\right| d \mu} \\
& \leq 2\left(\int_{\Omega} \frac{|\phi|^{2}}{|x|^{2}\left(\log \frac{a R}{|x|}\right)^{2}} d \mu\right)^{\frac{1}{2}}\left\|\nabla \phi+\frac{1}{2} \phi \frac{\nabla \rho}{\rho}\right\|_{L^{2}(\Omega ; d \mu)} .
\end{aligned}
$$

Therefore we have

$$
\frac{1}{4} \int_{\Omega} \frac{|\phi|^{2}}{|x|^{2}\left(\log \frac{a R}{|x|}\right)^{2}} d \mu \leq\left\|\nabla \phi+\frac{1}{2} \phi \frac{\nabla \rho}{\rho}\right\|_{L^{2}(\Omega ; d \mu)}^{2} .
$$


Note that $\rho \in C^{1, \alpha}(\Omega)$ for some $\alpha \in(0,1)$ by (H1) and the Sobolev embedding. Thus by applying the same argument as the proof of Proposition 3.1. in [8] with (H2), we obtain

$$
\begin{aligned}
\left\|\nabla \phi+\frac{1}{2} \phi \frac{\nabla \rho}{\rho}\right\|_{L^{2}(\Omega ; d \mu)}^{2} \leq\left[\frac { 4 } { 1 - 2 \varepsilon } \left(\frac{1}{4}\right.\right. & \left.\left.+\frac{\eta}{2}\right)+1+\frac{1}{2 \eta}\right] \int_{\Omega}|\nabla \phi|^{2} d \mu \\
& +\frac{C_{\varepsilon}(1+2 \eta)}{2(1-2 \varepsilon)} \int_{\Omega} \phi^{2} d \mu
\end{aligned}
$$

for each $\eta>0, \varepsilon \in\left(0, \frac{1}{2}\right)$ and a constant $C_{\varepsilon}>0$ in (H2). If we take $\eta=\frac{\sqrt{1-2 \varepsilon}}{2}$, then the function $\eta \mapsto \frac{4}{1-2 \varepsilon}\left(\frac{1}{4}+\frac{\eta}{2}\right)+1+\frac{1}{2 \eta}$ in (12) attains its minimum $\frac{2(1-\varepsilon+\sqrt{1-2 \varepsilon})}{1-2 \varepsilon}$, which goes to 4 from above as $\varepsilon \rightarrow 0$. Therefore, from (11) and (12), we get (10).

\section{Existence and nonexistence of positive solution}

In this section, we consider the following two dimensional Kolmogorov equation perturbed by a singular potential

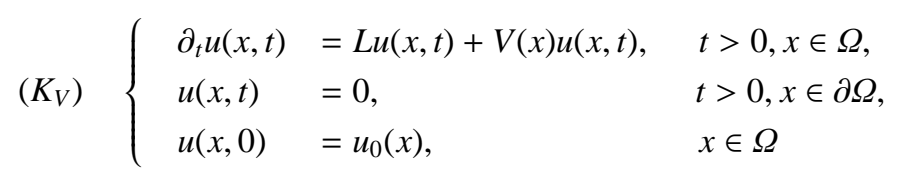

where $\Omega \subseteq \mathbb{R}^{2}$ is a domain, $0 \in \Omega, u_{0} \in L^{2}(\Omega ; d \mu), d \mu=\rho(x) d x$ is a probability Borel measure, $V \in L_{l o c}^{1}(\Omega), V \geq 0$, and $L$ is the Kolmogorov operator given by

$$
L u=\Delta u+\frac{\nabla \rho}{\rho} \cdot \nabla u
$$

Of course if $\Omega=\mathbb{R}^{2}$, we do not impose the Dirichlet boundary conditions. Especially, if $\rho(x)=\rho_{A}(x)=c \exp \left(-\frac{1}{2}\left(x^{t} A x\right)\right)$ and $A$ is a positive semi-definite real $2 \times 2$ symmetric matrix, then $L=L_{A}$ is the symmetric Ornstein-Uhlenbeck operator $L_{A} u=$ $\Delta u-A x \cdot \nabla u$. We define the bottom of the spectrum of $-(L+V)$ to be

$$
\lambda_{1}(L+V):=\inf _{0 \neq \phi \in H_{0}^{1}(\Omega ; d \mu)} \frac{\int_{\Omega}|\nabla \phi|^{2} d \mu-\int_{\Omega} V \phi^{2} d \mu}{\int_{\Omega} \phi^{2} d \mu} .
$$

We put the following definition.

Definition 1 We say that $u$ is a weak solution to $\left(K_{V}\right)$ if for each $T>0$ and any compact subset $K \subset \Omega$, we have $u \in C\left([0, T] ; L^{2}(\Omega ; d \mu)\right), V u \in L^{1}(K \times(0, T), d \mu d t)$ and

$$
\int_{0}^{T} \int_{\Omega} u\left(-\partial_{t} \phi-L \phi\right) d \mu d t-\int_{\Omega} u_{0} \phi(\cdot, 0) d \mu=\int_{0}^{T} \int_{\Omega} V u \phi d \mu d t
$$


for all $\phi \in W_{2}^{2,1}\left(Q_{T}\right)$ such that $\phi(\cdot, t)$ has compact support in $\Omega$ and $\phi(\cdot, T)=0$. Here $Q_{T}=\Omega \times(0, T)$ and $W_{2}^{2,1}\left(Q_{T}\right)$ denotes a standard parabolic Sobolev space:

$$
W_{2}^{2,1}\left(Q_{T}\right)=\left\{u \in L^{2}\left(Q_{T}\right): D_{x}^{\alpha} u \in L^{2}\left(Q_{T}\right) \text { for }|\alpha| \leq 2, \partial_{t} u \in L^{2}\left(Q_{T}\right)\right\} .
$$

Let $\Omega$ be a smooth bounded domain in $\mathbb{R}^{2}, 0 \in \Omega$, and $R=\sup _{x \in \Omega}|x|$. In this case, as in [4] and [8], we can obtain the existence and nonexistence result of solutions to $\left(K_{V}\right)$ as follows. Since the way of the proof is almost the same as [4] and [8], we give here the outline of the proof only.

Theorem 3 Assume that $0<\rho \in C^{1}(\Omega) \cap C(\bar{\Omega})$ and $0 \leq V \in L_{l o c}^{1}(\Omega)$. Then the following assertions hold:

(i) If $\lambda_{1}(L+V)>-\infty$, then for any $u_{0} \geq 0, u_{0} \in L^{2}(\Omega ; d \mu)$, there exists a positive weak solution $u \in C\left([0, \infty), L^{2}(\Omega ; d \mu)\right)$ of $\left(K_{V}\right)$ satisfying

$$
\|u(t)\|_{L^{2}(\Omega ; d \mu)} \leq M e^{\omega t}\left\|u_{0}\right\|_{L^{2}(\Omega ; d \mu)}, \quad t \geq 0
$$

for some constants $M \geq 1$ and $\omega \in \mathbb{R}$.

(ii) If $\lambda_{1}(L+V)=-\infty$, then for any $0 \leq u_{0} \in L^{2}(\Omega ; d \mu) \backslash\{0\}$, there is no positive weak solution of $\left(K_{V}\right)$ satisfying (13).

Proof of Theorem 3. (i) Assume $\lambda_{1}(L+V)>-\infty$ and take $u_{0} \geq 0, u_{0} \not \equiv 0$. Set $V_{n}(x)=\min \{V(x), n\}$ and $u_{0, n}(x)=\min \left\{u_{0}(x), n\right\}$. Note that since $\rho \in C(\bar{\Omega})$, the $L^{2}$ norm is equivalent to the $L_{\mu}^{2}$ norm. Consider the following truncated problem $\left(K_{V_{n}}\right)$ :

$$
\left(K_{V_{n}}\right)\left\{\begin{array}{llrl}
\partial_{t} u_{n}(x, t) & =L u_{n}(x, t)+V_{n}(x) u_{n}(x, t), & t>0, x \in \Omega, \\
u_{n}(x, t) & =0, & t>0, x \in \partial \Omega, \\
u_{n}(x, 0) & =u_{0, n}(x), & x \in \Omega .
\end{array}\right.
$$

Since $V_{n}$ and $u_{0, n}$ are bounded and nonnegative and the drift term $\frac{\nabla \rho}{\rho}$ is also bounded, $\left(K_{V_{n}}\right)$ admits a unique positive classical solution $u_{n}$, see e.g. Proposition C.3.2. in [15]. Furthermore $0<u_{n}(x, t) \leq u_{n+1}(x, t)$ for $n \in \mathbb{N}$ holds on $\Omega \times(0, \infty)$, see e.g. Proposition C.2.3. in [15]. If we multiply $\left(K_{V_{n}}\right)$ by $u_{n}$ and integrate by parts, we obtain the following in the same way as [8]:

$$
\left\|u_{n}(t)\right\|_{L^{2}(\Omega ; d \mu)} \leq e^{-\lambda_{1}(L+V) t}\left\|u_{0, n}\right\|_{L^{2}(\Omega ; d \mu)}, \quad t \geq 0,
$$

which yields that

$$
\left\|u_{n}(t)\right\|_{L^{2}(\Omega ; d \mu)} \leq e^{-\lambda_{1}(L+V) t}\left\|u_{0}\right\|_{L^{2}(\Omega ; d \mu)}, \quad t \geq 0 .
$$

By the monotone convergence theorem, we observe that $u_{n}(t)$ converges to $u(t)$ in $L^{2}(\Omega ; d \mu)$ uniformly for $t \in[0, T]$. Since $u_{n}$ is a weak solution of $\left(K_{V_{n}}\right)$, it follows that $u$ is a weak solution of $\left(K_{V}\right)$. The estimate (13) follows from (14) and it holds with $M=1$.

(ii) Assume $\lambda_{1}(L+V)=-\infty$ and assume that there exists a positive solution $u$ of $\left(K_{V}\right)$ with initial data $0 \leq u_{0} \in L^{2}(\Omega ; d \mu) \backslash\{0\}$ satisfying (13). We shall derive a 
contradiction. Fix $\phi \in C_{c}^{\infty}(\Omega)$ with $\int_{\Omega} \phi^{2} d x=1$. Let $u_{n}$ be the unique solution of $\left(K_{V_{n}}\right)$ and $v_{n}$ be the unique solution of

$$
\left(K_{n}\right) \quad\left\{\begin{array}{llr}
\partial_{t} v_{n}(x, t) & =L v_{n}(x, t), & t>0, x \in \Omega, \\
v_{n}(x, t) & =0, & t>0, x \in \partial \Omega, \\
v_{n}(x, 0) & =u_{0, n}(x), & x \in \Omega .
\end{array}\right.
$$

We observe that

$$
u(x, t) \geq u_{n}(x, t) \geq v_{n}(x, t) \geq v_{1}(x, t), \quad t \geq 0 .
$$

It is known that there exists a unique positive function $G_{\Omega} \in C((0, \infty) \times \Omega \times \Omega)$ such that for $u_{0, n} \in C(\bar{\Omega})$,

$$
v_{n}(t, x)=\int_{\Omega} G_{\Omega}(t, x, y) u_{0, n}(y) d y, \quad t>0, x \in \Omega,
$$

see e.g. Proposition C.3.2. in [15]. Since there exists a ball $B_{r}$ such that $u_{0,1}(x)>0$ for $x \in B_{r}$, we observe that for a.e. $x \in \operatorname{supp} \phi$,

$$
\begin{aligned}
v_{1}(t, x) & =\int_{\Omega} G_{\Omega}(t, x, y) u_{0,1}(y) d y \\
& \left.\geq \int_{(x, y) \in \operatorname{supp} \phi \times B_{r}} G_{\Omega}(t, x, y)\right) \int_{B_{r}} u_{0,1}(x) d x=: c_{r}\left(t ; u_{0,1}\right)>0 .
\end{aligned}
$$

Thus by (15), we have $u_{n}(x, t) \geq c_{r}\left(t ; u_{0,1}\right)>0$. If we multiply $\left(K_{V_{n}}\right)$ by $\frac{\phi^{2}}{u_{n}}$ and integrate by parts, then for every $t>1$ we obtain

$$
\int_{\Omega} V_{n} \phi^{2} d \mu \leq \partial_{t}\left(\int_{\Omega}\left(\log u_{n}(t)\right) \phi^{2} d \mu\right)+\int_{\Omega}|\nabla \phi|^{2} d \mu .
$$

By integrating from $t=1$ to $t=t$, we have

$$
(t-1) \int_{\Omega} V_{n} \phi^{2} d \mu \leq \int_{\Omega}\left(\log \frac{u_{n}(t)}{u_{n}(1)}\right) \phi^{2} d \mu+(t-1) \int_{\Omega}|\nabla \phi|^{2} d \mu
$$

for $t>1$ and any $n \in \mathbb{N}$, see [8]. Since there exists a minimal solution $\tilde{u}(t):=$ $\lim _{n \rightarrow \infty} u_{n}(t)$ by (15) and the monotone convergence theorem, we obtain

$$
\begin{aligned}
& \int_{\Omega} V \phi^{2} d \mu-\int_{\Omega}|\nabla \phi|^{2} d \mu \leq \frac{1}{(t-1)}\left[\int_{\Omega}(\log \tilde{u}(t)) \phi^{2} d \mu-\int_{\Omega}(\log \tilde{u}(1)) \phi^{2} d \mu\right] \\
& \leq \frac{1}{(t-1)}\left[\log \left(M\left\|u_{0}\right\|_{L^{2}(\Omega ; d \mu)}\right)+\omega t+\log \|\phi\|_{\infty}-\int_{\Omega}(\log \tilde{u}(1)) \phi^{2} d \mu\right] \leq C<\infty
\end{aligned}
$$

in the same way as [8]. This contradicts the assumption $\lambda_{1}(L+V)=-\infty$. Therefore there is no positive weak solution of $\left(K_{V}\right)$ satisfying (13).

As a consequence of Theorem 3 and Remark 1, we obtain the main result. 
Theorem 4 Let $\Omega$ be a bounded domain in $\mathbb{R}^{2}, 0 \in \Omega, a \geq 1$, and $R=\sup _{x \in \Omega}|x|$. Assume that $A$ be a positive semi-definite real $2 \times 2$-symmetric matrix and $0 \leq V(x) \leq$ $\frac{c}{|x|^{2}\left(\log \frac{a R}{|x|}\right)^{2}}$. Then the followings hold:

of

(i) If $0 \leq c \leq \frac{1}{4}$, then there exists a positive weak solution $u \in C\left([0, \infty), L^{2}\left(\Omega ; d \mu_{A}\right)\right)$

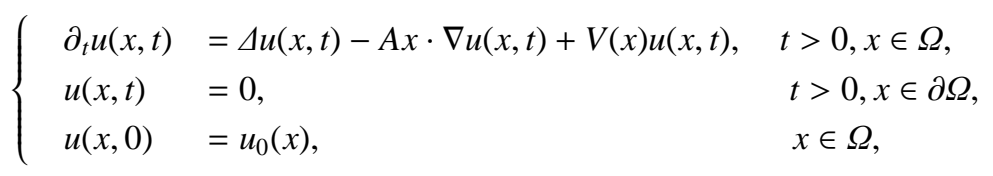

satisfying

$$
\|u(t)\|_{L^{2}\left(\Omega ; d \mu_{A}\right)} \leq M e^{\omega t}\left\|u_{0}\right\|_{L^{2}\left(\Omega ; d \mu_{A}\right)}, \quad t \geq 0
$$

for some constants $M \geq 1, \omega \in \mathbb{R}$, and any $0 \leq u_{0} \in L^{2}\left(\Omega ; d \mu_{A}\right)$.

(ii) If $c>\frac{1}{4}$, then for any $0 \leq u_{0} \in L^{2}\left(\Omega ; d \mu_{A}\right) \backslash\{0\}$, there is no positive weak solution of (16) with $V(x)=\frac{c}{|x|^{2}\left(\log \frac{a R}{|x|}\right)^{2}}$ satisfying (17).

Furthermore the following result also follows from Proposition 1 and Theorem 3.

Corollary 1 Let $\Omega$ be a bounded domain in $\mathbb{R}^{2}, 0 \in \Omega, a \geq 1$, and $R=\sup _{x \in \Omega}|x|$. Assume that $(H 1)-(H 2)$ in $\S 2$ are satisfied and $0 \leq V(x) \leq \frac{c}{|x|^{2}\left(\left.\log \frac{a R}{|x|}\right|^{2}\right.}$. If $c<\frac{1}{16}$, then there exists a weak solution $u \in C\left([0, \infty), L^{2}(\Omega ; d \mu)\right)$ of $\left(K_{V}\right)$ satisfying

$$
\|u(t)\|_{L^{2}(\Omega ; d \mu)} \leq M e^{\omega t}\left\|u_{0}\right\|_{L^{2}(\Omega ; d \mu)}, \quad t \geq 0
$$

for some constants $M \geq 1, \omega \in \mathbb{R}$, and any $0 \leq u_{0} \in L^{2}(\Omega ; d \mu)$.

\section{Weighted Hardy inequality on the half space}

In this section, we obtain a weighted $L^{p}$-Hardy inequality on the half space $\mathbb{R}_{+}^{N}=$ $\left\{x=\left(x^{\prime}, x_{N}\right) \in \mathbb{R}^{N-1} \times \mathbb{R} \mid x_{N}>0\right\}$. Though the obtained inequality does not have any concrete application in this paper, we hope it may be also useful to the study of the corresponding parabolic problems.

Theorem 5 Let $1<p<\infty$, let A be a real $N \times N$-symmetric positive semi-definite matrix, and let

$$
d \mu_{A}=\rho_{A}(x) d x \quad \text { with } \quad \rho_{A}(x)=c \cdot \exp \left(-\frac{1}{p}\left(x^{t} A x\right)^{\frac{p}{2}}\right), x \in \mathbb{R}_{+}^{N},
$$

where $c$ is chosen so that $\int_{\mathbb{R}_{+}^{N}} \rho_{A} d x=1$. Then the inequality

$$
\left(\frac{p-1}{p}\right)^{p} \int_{\mathbb{R}_{+}^{N}} \frac{|u|^{p}}{x_{N}^{p}} d \mu_{A} \leq \int_{\mathbb{R}_{+}^{N}}\left|\frac{\partial u}{\partial x_{N}}\right|^{p} d \mu_{A}-\left(\frac{p-1}{p}\right)^{p-1} \int_{\mathbb{R}_{+}^{N}} \frac{|u|^{p}\left(x^{t} A x\right)^{\frac{p-2}{2}}(A x)_{N}}{x_{N}^{p-1}} d \mu_{A}
$$

holds for all $u \in W_{\mu_{A}, 0}^{1, p}\left(\mathbb{R}_{+}^{N}\right)$. Moreover the constant $\left(\frac{p-1}{p}\right)^{p}$ in the left-hand side of (19) is optimal. 
Proof of Theorem 5. It is enough to show that the inequality (19) holds for all $u \in$ $C_{c}^{1}\left(\mathbb{R}_{+}^{N}\right)$. We fix $\lambda \geq 0$ which will be chosen later. Set

$$
\mathbf{F}(x)=\left(0, \cdots, 0, \lambda \rho_{A}(x) x_{N}^{1-p}\right) \quad \text { for } x \in \mathbb{R}_{+}^{N} .
$$

Then we compute that

$$
\operatorname{div} \mathbf{F}(x)=\frac{\partial}{\partial x_{N}}\left(\lambda \rho_{A}(x) x_{N}^{1-p}\right)=-\lambda \rho_{A}(x)\left[\frac{p-1}{x_{N}^{p}}+\frac{\left(x^{t} A x\right)^{\frac{p-2}{2}}(A x)_{N}}{x_{N}^{p-1}}\right] .
$$

By applying integration by parts and Young's inequality, we have

$$
\begin{aligned}
& \int_{\mathbb{R}_{+}^{N}}|u|^{p} \lambda\left[\frac{p-1}{x_{N}^{p}}+\frac{\left(x^{t} A x\right)^{\frac{p-2}{2}}(A x)_{N}}{x_{N}^{p-1}}\right] \rho_{A}(x) d x \\
& =-p \int_{\mathbb{R}_{+}^{N}}|u|^{p-2} u(\nabla u \cdot \mathbf{F}) d x \\
& =-p \lambda \int_{\mathbb{R}_{+}^{N}} \frac{|u|^{p-2} u}{x_{N}^{p-1}}\left(\frac{\partial u}{\partial x_{N}}\right) d \mu_{A} \\
& \leq \int_{\mathbb{R}_{+}^{N}}\left|\frac{\partial u}{\partial x_{N}}\right|^{p} d \mu_{A}+(p-1) \lambda^{\frac{p}{p-1}} \int_{\mathbb{R}_{+}^{N}} \frac{|u|^{p}}{x_{N}^{p}} d \mu_{A},
\end{aligned}
$$

which yields that

$$
(p-1)\left(\lambda-\lambda^{\frac{p}{p-1}}\right) \int_{\mathbb{R}_{+}^{N}} \frac{|u|^{p}}{x_{N}^{p}} d \mu_{A} \leq \int_{\mathbb{R}_{+}^{N}}\left|\frac{\partial u}{\partial x_{N}}\right|^{p} d \mu_{A}-\lambda \int_{\mathbb{R}_{+}^{N}} \frac{|u|^{p}\left(x^{t} A x\right)^{\frac{p-2}{2}}(A x)_{N}}{x_{N}^{p-1}} d \mu_{A} .
$$

If we choose $\lambda=\left(\frac{p-1}{p}\right)^{p-1}$ which attains the maximum of the function $\lambda \mapsto(\lambda-$ $\left.\lambda^{\frac{p}{p-1}}\right)$ on the half line $[0, \infty)$, then we obtain the inequality (19) for all $u \in C_{c}^{1}\left(\mathbb{R}_{+}^{N}\right)$. Therefore the inequality (19) also holds for all $u \in W_{\mu_{A}, 0}^{1, N}\left(\mathbb{R}_{+}^{N}\right)$ by density.

Next we show the optimality of the constant $\left(\frac{p-1}{p}\right)^{p}$ in (19). To do so, we fix $\lambda>\left(\frac{p-1}{p}\right)^{p}$ and take any $\tilde{\lambda} \in \mathbb{R}$. Set

$$
\begin{aligned}
& E(u)=\int_{\mathbb{R}_{+}^{N}}\left|\frac{\partial u}{\partial x_{N}}\right|^{p} d \mu_{A}-\tilde{\lambda} \int_{\mathbb{R}_{+}^{N}} \frac{|u|^{p}\left(x^{t} A x\right)^{\frac{p-2}{2}}(A x)_{N}}{x_{N}^{p-1}} d \mu_{A}, \\
& F_{\lambda}(u)=\lambda \int_{\mathbb{R}_{+}^{N}} \frac{|u|^{p}}{x_{N}^{p}} d \mu_{A}
\end{aligned}
$$

for $u \in W_{\mu_{A}, 0}^{1, N}\left(\mathbb{R}_{+}^{N}\right) \backslash\{0\}$. Now we consider a test function $\varphi_{\gamma, \varepsilon} \in W_{\mu_{A}, 0}^{1, p}\left(\mathbb{R}_{+}^{N}\right)$ given by

$$
\varphi_{\gamma, \varepsilon}(x)=x_{N}^{\gamma} \xi_{\varepsilon}\left(x_{N}\right) \xi_{\varepsilon}\left(\left|x^{\prime}\right|\right)
$$


where $x=\left(x^{\prime}, x_{N}\right) \in \mathbb{R}^{N-1} \times \mathbb{R}_{+}, \gamma>\frac{p-1}{p}, \varepsilon>0$ will be chosen later independent of $\gamma$, and $\xi_{\varepsilon}$ is a cut-off function defined by

$$
\xi_{\varepsilon}(t)= \begin{cases}1, & \text { if } 0 \leq t \leq \frac{\varepsilon}{2} \\ \frac{2}{3 \varepsilon}(2 \varepsilon-t), & \text { if } \frac{\varepsilon}{2}<t<2 \varepsilon \\ 0, & \text { if } t \geq 2 \varepsilon\end{cases}
$$

Note that there exists $\alpha_{2}>0$ such that $0 \leq x^{t} A x \leq \alpha_{2}|x|^{2}$ for all $x \in \mathbb{R}_{+}^{N}$. Then we have

$$
\begin{aligned}
F_{\lambda}\left(\varphi_{\gamma, \varepsilon}\right) & \geq \lambda c \int_{x_{N}=0}^{\frac{\varepsilon}{2}} \int_{\left|x^{\prime}\right| \leq \frac{\varepsilon}{2}} x_{N}^{\gamma p-p} \exp \left(-\frac{\alpha_{2}^{\frac{p}{2}}}{p}|x|^{p}\right) d x^{\prime} d x_{N} \\
& +\lambda c \int_{x_{N}=0}^{\frac{\varepsilon}{2}} \int_{\frac{\varepsilon}{2} \leq\left|x^{\prime}\right| \leq 2 \varepsilon} x_{N}^{\gamma p-p} \xi_{\varepsilon}\left(\left|x^{\prime}\right|\right)^{p} \exp \left(-\frac{\alpha_{2}^{\frac{p}{2}}}{p}|x|^{p}\right) d x^{\prime} d x_{N}
\end{aligned}
$$

which yields that

$$
\begin{aligned}
F_{\lambda}\left(\varphi_{\gamma, \varepsilon}\right) & \geq \lambda \frac{c\left|B^{N-1}\left(\frac{\varepsilon}{2}\right)\right|}{\gamma p-p+1}\left(\frac{\varepsilon}{2}\right)^{\gamma p-p+1} \exp \left(-\frac{\alpha_{2}^{\frac{p}{2}}}{p}\left(\frac{\varepsilon}{\sqrt{2}}\right)^{p}\right) \\
& +\lambda \frac{c C(\varepsilon)}{\gamma p-p+1}\left(\frac{\varepsilon}{2}\right)^{\gamma p-p+1} \exp \left(-\frac{\alpha_{2}^{\frac{p}{2}}}{p}\left(\frac{3}{2} \varepsilon\right)^{p}\right) .
\end{aligned}
$$

Here $\left|B^{N-1}(r)\right|=\int_{\left|x^{\prime}\right| \leq r} d x^{\prime}$ denotes the volume of the $(N-1)$-dimensional ball with radius $r$, and

$$
C(\varepsilon)=\int_{\frac{\varepsilon}{2} \leq\left|x^{\prime}\right| \leq 2 \varepsilon} \xi_{\varepsilon}\left(\left|x^{\prime}\right|\right)^{p} d x^{\prime} .
$$

On the other hand, we obtain

$$
\begin{aligned}
\int_{\mathbb{R}_{+}^{N}}\left|\frac{\partial \varphi_{\gamma, \varepsilon}}{\partial x_{N}}\right|^{p} d \mu_{A} & \leq \gamma^{p} c \int_{x_{N}=0}^{\frac{\varepsilon}{2}} \int_{\left|x^{\prime}\right| \leq \frac{\varepsilon}{2}} x_{N}^{\gamma p-p} d x^{\prime} d x_{N} \\
& +\gamma^{p} c \int_{x_{N}=0}^{\frac{\varepsilon}{2}} \int_{\frac{\varepsilon}{2} \leq\left|x^{\prime}\right| \leq 2 \varepsilon} x_{N}^{\gamma p-p} \xi_{\varepsilon}\left(\left|x^{\prime}\right|\right)^{p} d x^{\prime} d x_{N} \\
& +c \int_{x_{N}=\frac{\varepsilon}{2}}^{2 \varepsilon} \int_{\left|x^{\prime}\right| \leq 2 \varepsilon}\left|\frac{\partial}{\partial x_{N}}\left(x_{N}^{\gamma} \xi_{\varepsilon}\left(x_{N}\right)\right)\right|^{p} \xi_{\varepsilon}\left(\left|x^{\prime}\right|\right)^{p} d x^{\prime} d x_{N}
\end{aligned}
$$

which yields that

$$
\begin{aligned}
\int_{\mathbb{R}_{+}^{N}}\left|\frac{\partial \varphi_{\gamma, \varepsilon}}{\partial x_{N}}\right|^{p} d \mu_{A} & \leq \gamma^{p} \frac{c\left|B^{N-1}\left(\frac{\varepsilon}{2}\right)\right|}{\gamma p-p+1}\left(\frac{\varepsilon}{2}\right)^{\gamma p-p+1}+\gamma^{p} \frac{c C(\varepsilon)}{\gamma p-p+1}\left(\frac{\varepsilon}{2}\right)^{\gamma p-p+1} \\
& +c 2^{p-1}\left(\gamma^{p} D(\varepsilon)+E(\varepsilon)\right)
\end{aligned}
$$


where

$$
\begin{aligned}
& D(\varepsilon)=\int_{x_{N}=\frac{\varepsilon}{2}}^{2 \varepsilon} \int_{\left|x^{\prime}\right| \leq 2 \varepsilon} x_{N}^{\gamma p-p} \xi_{\varepsilon}\left(x_{N}\right)^{p} \xi_{\varepsilon}\left(\left|x^{\prime}\right|\right)^{p} d x^{\prime} d x_{N}, \\
& E(\varepsilon)=\int_{x_{N}=\frac{\varepsilon}{2}}^{2 \varepsilon} \int_{\left|x^{\prime}\right| \leq 2 \varepsilon} x_{N}^{\gamma p}\left(\frac{2}{3 \varepsilon}\right)^{p} \xi_{\varepsilon}\left(\left|x^{\prime}\right|\right)^{p} d x^{\prime} d x_{N} .
\end{aligned}
$$

Note that

$$
\begin{aligned}
D(\varepsilon) & \leq\left|B^{N-1}(2 \varepsilon)\right| \int_{x_{N}=\frac{\varepsilon}{2}}^{2 \varepsilon} x_{N}^{\gamma p-p} \xi_{\varepsilon}\left(x_{N}\right)^{p} d x_{N} \\
& \leq C \varepsilon^{N-1} \int_{t=\frac{\varepsilon}{2}}^{2 \varepsilon} t^{\gamma p-p} d t \\
& =C \varepsilon^{\gamma p-p+N}
\end{aligned}
$$

and

$$
E(\varepsilon) \leq\left|B^{N-1}(2 \varepsilon)\right| \int_{x_{N}=\frac{\varepsilon}{2}}^{2 \varepsilon} x_{N}^{\gamma p}\left(\frac{2}{3 \varepsilon}\right)^{p} d x_{N}=C \varepsilon^{\gamma p-p+N}
$$

for some absolute value $C>0$. In the same way as above, we also obtain

$$
\begin{aligned}
\left|\int_{\mathbb{R}_{+}^{N}} \frac{\left|\varphi_{\gamma, \varepsilon}\right|^{p}\left(x^{t} A x\right)^{\frac{p-2}{2}}(A x)_{N}}{x_{N}^{p-1}} d \mu_{A}\right| & \leq \alpha_{2}^{\frac{p-2}{2}} \int_{x_{N}=0}^{2 \varepsilon} \int_{\left|x^{\prime}\right| \leq 2 \varepsilon} x_{N}^{\gamma p-p+1}|A||x|^{p-1} \rho_{A}(x) d x \\
& \leq C \varepsilon^{\gamma p+N}
\end{aligned}
$$

for $\gamma>\frac{p-1}{p}$ sufficiently close to $\frac{p-1}{p}$. From (21), (22), (23), and (24), we have

$$
E\left(\varphi_{\gamma, \varepsilon}\right) \leq \gamma^{p} \frac{c\left|B^{N-1}\left(\frac{\varepsilon}{2}\right)\right|}{\gamma p-p+1}\left(\frac{\varepsilon}{2}\right)^{\gamma p-p+1}+\gamma^{p} \frac{c C(\varepsilon)}{\gamma p-p+1}\left(\frac{\varepsilon}{2}\right)^{\gamma p-p+1}+C
$$

for an absolute value $C>0$ when $\gamma>\frac{p-1}{p}$ and $\varepsilon \in(0,1)$.

We have chosen a small $\varepsilon>0$ in advance such that

$$
\lambda \exp \left(-\frac{\alpha_{2}^{\frac{p}{2}}}{p}\left(\frac{3}{2} \varepsilon\right)^{p}\right)>\left(\frac{p-1}{p}\right)^{p},
$$

which is possible since $\lambda>\left(\frac{p-1}{p}\right)^{p}$. For this choice of $\varepsilon$, we may take $\gamma>\frac{p-1}{p}$ sufficiently close to realize that

$$
\lambda \exp \left(-\frac{\alpha_{2}^{\frac{p}{2}}}{p}\left(\frac{\varepsilon}{\sqrt{2}}\right)^{p}\right)>\lambda \exp \left(-\frac{\alpha_{2}^{\frac{p}{2}}}{p}\left(\frac{3}{2} \varepsilon\right)^{p}\right)>\gamma^{p}
$$

holds true. From the estimates (20) and (25), we observe that

$$
E\left(\varphi_{\gamma, \varepsilon}\right)<F_{\lambda}\left(\varphi_{\gamma, \varepsilon}\right)
$$


for $\gamma>\frac{p-1}{p}$ sufficiently close to $\frac{p-1}{p}$ satisfying (26). Therefore the inequality (19) never holds if the constant on the left-hand side of (19) is larger than $\left(\frac{p-1}{p}\right)^{p}$. Hence the constant $\left(\frac{p-1}{p}\right)^{p}$ in (19) is optimal.

Acknowledgements The authors thank the anonymous referees for their careful reading the manuscript. Part of this work was supported by JSPS Grant-in-Aid for Fellows(DC2), No.16J07472 (M.S), and JSPS Grant-in-Aid for Scientific Research (B), No.15H03631,

\section{References}

1. Adimurthi, Sandeep, K., Existence and non-existence of the first eigenvalue of the perturbed HardySobolev operator, Proc. Roy. Soc. Edinburgh Sect. A 132 (2002), no.5, 1021-1043.

2. Arendt, W., Goldstein, G. R., Goldstein, J. A., Outgrowths of Hardy's inequality, Recent advances in differential equations and mathematical physics, 51-68, Contemp. Math., 412, Amer. Math. Soc., Providence, RI, 2006.

3. Baras, P., Goldstein, J. A., The heat equation with a singular potential, Trans. Amer. Math. Soc., 284 (1984), 121-139.

4. Cabré, X., and Martel, Y., Existence versus explosion instantanée pour des équations de la chaleur linéaires avec potential singulier, C. R. Acad. Sci. Paris Sér. I Math. 329 (1999), 973-978.

5. Goldstein, J. A., Kombe, I., Instantaneous blow up, Contemp. Math. 327 (2003), 141-150.

6. Goldstein, J. A., Kombe, I., Gaussian estimates and instantaneous blow up, Positivity. 12 (2008), 75-82.

7. Goldstein, G. R., Goldstein, J. A., Rhandi, A., Kolmogorov equations perturbed by an inverse-square potential, Discrete Contin. Dyn. Syst. Ser. S 4 (2011), no. 3, 623-630.

8. Goldstein, G. R., Goldstein, J. A., Rhandi, A., Weighted Hardy's inequality and the Kolmogorov equation perturbed by an inverse-square potential, Appl. Anal. 91 (2012), no. 11, 2057-2071.

9. Goldstein, J. A., Hauer, D., Rhandi, A., Existence and nonexistence of positive solutions of pKolmogorov equations perturbed by a Hardy potential, Nonlinear Anal. 131 (2016), 121-154.

10. Hauer, D., Rhandi, A., A weighted Hardy inequality and nonexistence of positive solutions, Arch. Math., 100 (2013), 273-287.

11. Goldstein, J. A., Zhang, Q. S., On a degenerate heat equation with a singular potential, J. Funct. Anal. 186 (2001), 342-359.

12. Goldstein, J. A., Zhang, Q. S., Linear parabolic equations with strong singular potentials, Trans. Amer. Math. Soc. 355 (2003), 197-211.

13. Ioku, N., Ishiwata, M., A scale invariant form of a critical Hardy inequality, Int. Math. Res. Not. IMRN, (2015), no. 18, 8830-8846.

14. Leray, Jean., Étude de diverses équations intégrales non linéaires et de quelquesproblèmes que pose lhydrodynamique. Thesis (French) (1933). $82 \mathrm{pp}$.

15. Lorenzi, L., Bertoldi, M., Analytical methods for Markov semigroups, Pure and Applied Mathematics (Boca Raton), 283. Chapman \& Hall/CRC, Boca Raton, FL, 2007.

16. Sano, M., Scaling invariant Hardy type inequalities with non-standard remainder terms, Math. Inequal. Appl. 21 (2018), no. 1, 77-90.

17. Sano, M., Takahashi, F., Sublinear eigenvalue problems with singular weights related to the critical Hardy inequality, Electron. J. Diff. Equ., Vol. 2016 (2016), No. 212, pp. 1-12.

18. Sano, M., and Takahashi, F., Scale invariance structures of the critical and the subcritical Hardy inequalities and their improvements, Calc. Var. Partial Differential Equations., Vol. 56 (2017), No. 3 , Art. 69, 14 pages.

19. Takahashi, F., A simple proof of Hardy's inequality in a limiting case, Arch. Math., 104 (2015), no.1, $77-82$. 